\title{
A Gesture Interface Description Language for a Unified Gesture Platform
}

\author{
Geun-Hyung Kim1), Eunji Song2)
}

\begin{abstract}
Nowadays, the advent of smart devices equipped with the latest input technologies has changed the way users interact with smart devices. The gesture based user interface, as the natural user interface technologies, has attracted a lot of attention from researchers and developers. Gestures can be constituted in different ways; touching a screen, moving a pointing device, or making hand or body movements in a three-dimensional (3D) space. The various gesture input devices make application developers to maintain multiple source code families for the same applications adapting different gesture input devices. In this paper, we defined the gesture interface markup language (GIML) based on extensible markup language (XML) to describe gestures independently of the input devices. It also provides constraints necessary to determine which gesture has occurred and information required when UGesture platform interact with the gesture based application. The proposed GIML is based on our previous implemented the UGesture platform and the evaluation results, and so the GIML can be used to define new gestures for the UGesture platform and support new input hardwares.
\end{abstract}

Keywords : Gesture interface markup language, Unified gesture platform, 3D hand gesture, 3D hand movement detector

\section{Introduction}

Recently, various input devices have been released that allows users to interact in different ways with computing systems, where these new input devices allow diverse gestures to be employed. However, current user interface (UI) frameworks are still constraint in the observer patterns where events happen atomically and sequentially in time and the events are notified through callback messages. Besides, different gesture handling mechanisms in the UI programming interface frameworks have provided the same paradigm by hiding the gesture

Received(March 12, 2018), Review Result(1st: March 26, 2018, 2nd: April 23, 2018), Accepted(April 30, 2018)

${ }^{1}$ (Corresponding Author)Game Engineering Major, Dong-eui Univ., 176, Eomgwang-ro, Busanjin-gu, Busan, 47340 , Korea email: geunkim@deu.ac.kr

${ }^{2}$ Dept. of Digital Media Engineering, Dong-eui Univ., 176, Eomgwang-ro, Busanjin-gu, Busan, 47340, Korea email: sej1272@naver.com

*This research was supported by Basic Science Research Program through the National Research Foundation of Korea(NRF) funded by the Korea government (MOE: Ministry of Education): (NRF-2017R1D1A1B03035074 and NRF-2010-0025069) 
recognition logic. Thereby, generating high-level gesture events when a user's gesture is done and providing intermediate feedback to the handling mechanisms for low-level events, which are decoupled from high-level events, are important[1]. In order to allow the gesture recognition logic to support newly defined gesture, programmers should be able to hook low-level events came from the input sensors and to conduct the gesture recognition algorithm.

Users interact with computing systems though the UIs to control and operate it in an effective manner. Uls affect greatly on the system performance how well or how fast we do our works with applications behind it. UIs play an important role in the whole user experience of the devices of which it is part[2]. The authors in[2] have classified the major interaction styles into conversational, manipulative and explorative interactions in terms of the application usage style. Conversational interactions between humans and computing systems emulates humans' communication like discussions or dialogues. Voice recognition is an important technology that can enhance the conversational interactions. Manipulative interaction allows users to manipulate the items on the screen that can be used to make the behavior of UI dynamically. Explorative interaction is used in the applications where users have to explore the world behind the UI. These interactions can be used in an integrated form in an application, according to the applications.

UIs have evolved continuously along with computing systems, as various applications and usage styles have been developed. UIs have been classified in generations which align to the advent of the input hardwares according to Nielsen[2][3]. The command line interface (CLI) was an example of a first-generation UI, which mainly used a keyboard to put inputs and a screen to display outputs, for interactions to the computing system. The graphical user interface (GUI), a second-generation UI, allowed users to interact with computing systems through secondary interactions, such as icons and input fields opposing to CLIs. In GUIs, users were encouraged to find UI components, such as icons, pop-ups or dialog menus, on a screen. So the pointing devices were introduced as a way to interact with UI components on the GUIs. Most sophisticated and direct interaction devices became available after the development of GUIs, such as touch screen and pointing devices. GUIs have relied on both manipulational interactions and exploratory interactions[2]. The natural user interface (NUI) were introduced as the next generation UI which could support a user's cognition and interact with computing systems in a natural manner, without any interaction devices such as keyboards, pointing devices, or touch devices. The examples of natural Interaction devices for users are sensors, cameras, eye tracking, or wearable equipments, etc.

Currently, various device have been emerged and most users have owned one or more 
devices, including tablet PCs, smart phones and TVs, and so on. In addition, users want to keep similar service experiences when interacting with devices of different types. Owing to recent advances in Web technologies such as Hyper Text Markup Language 5 (HTML5) and responsive web, application developers have been able to provide the adaptive applications regardless of device types. Therefore, application developers have to think of various UIs for better user experience, when developing web applications for different devices, because of the differences in screen sizes, UI technologies, and corresponding event types among various devices. Thus, several gesture libraries, to resolve problem, have been proposed[4][5]. Because many types of input device have been developing and each device had its own device-specific application programming interfaces (APIs), it has been much difficult for application developers to support new devices directly as more devices have emerged on the market. Eventually operating systems offered a unified single API, which shields the application developers from the underlying device-specific APIs[6].

Since gesture interface devices investigate the movement expansion in time and space as the arguments for the interaction, compositional representation of different lower-level events into a higher-level gesture event is required. So a declarative description of the sequence of lower-level events or gestures enables it possible that the device senses for recognizing a custom gesture, raising a single specific gesture event when the movement completes[1]. Construction of consistent gestures for a wide variety of input hardwares (e.g., tangible objects, touch surfaces, body tracking, voice, brain-wave) is a very important issue. In this paper, we described the UGesture platform, which gives a unified handling mechanisms for different input devices, especially mouse, touch screen, touch pointer, and three-dimensional(3D) hand movement detector, including that employ sensors. We also define an extensible markup language (XML) based gesture interface markup language (GIML) that enables the UI designers to describe UIs and UI developers to implement the gesture handling functionality regardless of UI hardwares.

The rest of this paper is constituted as follows. In Section 2, we reviewed the related works. And then, we described the key functions of the UGesture platform in Section 3. In Section 4, we described the GIML schema and the gesture description example using GIML and we gave an example of enhancement in UGesture against Hammer library in Section 5. Finally, we gave our conclusions in Section 6. 


\section{Related Works}

UI technologies have evolved as long as computing systems have existed even before the human-computing system interaction (HCI) was established. The UIs namely were be understood as two directional interfaces that provide both inputs and outputs. In the beginning, the input and output interfaces were clearly separated but technological evolution has made these more closer to each other[2].

The UIs for early batch computing systems consisted of punched cards or equivalent media like paper tape as input devices and line printers as output devices. So, the UI for batch computing systems did not allow users to interact with the batch computing systems in real time. Since software was designed to make the best use of the processor, complicated UIs were considered and command-line interfaces (CLIs) were introduced in the mid-1960s. CLIs were closely associated with the rise of time-sharing computing systems. The latency of CLIs greatly dropped from days or hours to seconds, since the interaction between users and computing system consists of a series of request-response transactions[2]. This transaction model allowed users to receive response immediately after requests expressed as a texture commands in a specialized vocabulary typed using a keyboard.

The next core progression in UI came from the advance of video display terminals, graphic icons, and pointing devices to interact computing systems. This UI progression could enable an attractive user experience. The first GUI, developed in the 1970's, began the succession of computer graphic innovations on GUIs and conducted to where we are today. In today, most operating systems (OSs) have a windows-based system incorporating features[7].

Because of the increasing in popularity of the smart devices at the end of the 2000's, the UI design has started to change significantly[7]. The best dedicated UI for handheld devices was considered a touch screen GUI with multi-point touch functionalities that enabled users to interact with applications in new ways. With the advent of multi-touch UIs, handheld UIs have been designed around app functionality and these touch-based UIs might influence not only the UIs of desktop and laptop but also kiosks, ATMs, appliances, etc.

Along with mouse devices, keyboards, and touch screens, voice UIs such as Amazon echo and Google assistants are currently used in desktops, mobile devices, and embedded systems. Such functionalities are likely to become more prevalent in the future along with other UI advancements such as brain signal interface, virtual reality, augmented reality, or gesture functionality[7]. 
Since various devices may also support the same gestures regardless of device types, application developers should handle the discrepancies in gestures among devices when developing the applications which are able to run on several different devices. To consider the differences among touch gestures, Vilamore et el.[8] classified touch gestures as core gestures, basic operations, operations related to an object, and navigation operations. In the classification proposed by Vilamore et el.[8], core gesture consists of flick, pinch, drag, spread, tap, double tap, press, press and drag, press and tap, and rotate. In real situation, all devices do not supply whole core gestures. The gesture name may also be defined in different ways on different devices, though the gesture may keep the same meaning and movement.

For a web page on iOS, the gestures handled by Safari, such as a double tap to zoom and flick to pan, emulate mouse events[9]. Application developers could register to allow iOS-specific multi-touch gesture events directly. In addition, application developers can release the default Safari on iOS behavior and describe their own multi-touch and gesture events. The one finger event flows or two fingers gesture depends on if the selected elements are touchable or operable. To handle multi-touch and gesture events, application developers can use JavaScript DOM touch event classes available on iOS, which define three events; touchstart, touchmove, and touchend.

The pointer events specification of $\mathrm{W} 3 \mathrm{C}$ defined events and related interfaces for handling hardware-agnostic pointer inputs from devices including a mouse, pen, and touchscreen[10] which also described a mapping to fire mouse events for other pointer device types, thereby allowing compatibility with existing mouse based content.

To develop web applications, application developers have used web-based gesture libraries that execute with various devices, regardless of the event types occurred by the input devices. In general, users typically require to employ the web applications with the same gestures on any devices. The HammerJS[11] was proposed to provide the common gesture APIs for touch, mouse and pointer input devices. It defined the APIs for several multi-touch gestures including touch gestures. The HammerJS utilized corresponding object to handle each gesture respectively and the global management object that assembled event data, triggered corresponding gesture events and invokeed an event handler. However, The HammerJS only supports a mouse, pen, and touchscreen, whereas it lacks the 3D motion detectors[12].

\section{UGesture Platform}

Conventional gesture platforms have been classified into two groups: the one is to provide 
common touch gesture APIs for the handheld devices, and the other is the common pointing gesture APIs to support both a mouse and a touch screen. In this section, we describe the key functionalities of the unified gesture handling platform, that we have already developed to provide to unified gesture APIs for a touch screen, a 3D motion detector, and a mouse.

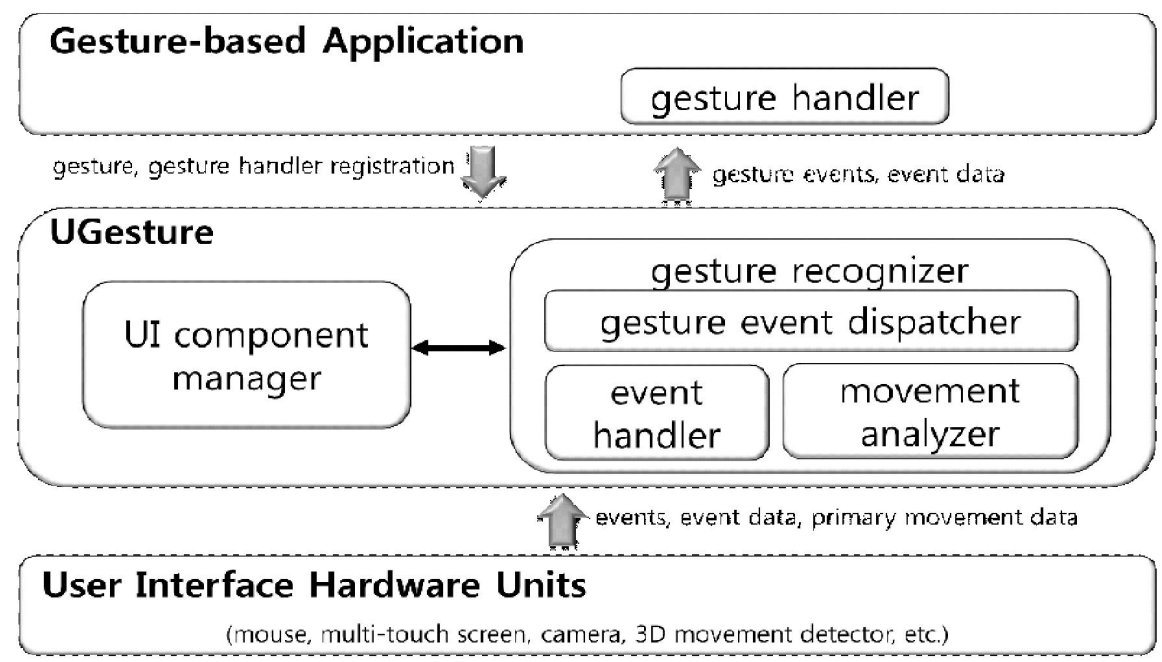

[Fig. 1] The functional architecture of the UGesture platform

Fig. 1 gives the functional architecture of gesture platform and its interactions among functionalities in the same layers or other layers. The UGesture platform encapsulates the underlying UI hardware devices and hides it from gesture-based applications. Gesture-based application developers can develop applications easily by using the UGesture platform regardless of the input device types.

Application developers register the required gestures and corresponding gesture handlers to the UGesture platform. Then, the platform invokes the corresponding gesture handler with related data to the specified gesture. The UGesture platform mainly consists of a UI component manager and a gesture recognizer.

The UI component manager maintains the binding information between a UI component and registered gestures over the UI component as well as the geographical location information of UI components. In addition, the UI component manager interacts with the gesture recognizer to check whether the specific gesture occurs on a given UI component.

The gesture recognizer consists of the event handler, the movement analyzer, and the gesture event dispatcher. The event handlers handles UI input device events, which may correspond to part of the specific gesture. The movement analyzer has the role of analyzing the movement 
data retrieved from UI hardware units and checking if the movement sequences correspond to the specific gesture. When the movement analyzer detects the gesture, it interacts with the gesture event dispatcher for generating the specific gesture event for the application.

\section{Gesture Interface Markup Language}

In order to describe the gestures formally, we investigated the relation among movement, action, gesture, and context as shown in Fig. 2. We defined the movement as the physical position or posture change of one or more objects and the action as a goal-directed movement or manipulation, and things done to achieve a particular purpose. We defined the gesture as a sequence of action(s) of body, especially hands and arms, that shows or emphasizes intentions. The same gesture can be interpreted differently according to the context, which is the situation underlying the relationship between gestures and meanings.

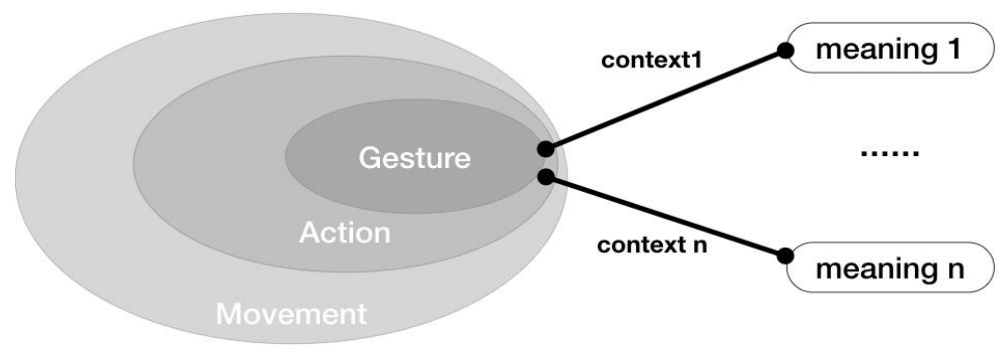

[Fig. 2] The relationship among gesture, action, movement, and meaning

To describe gestures using GIML, we considered 14 gestures; left, right, up, down, forward, backward, clockwise rotation, counter-clockwise rotation, tap, displace, double-tap, horizontal wave, and vertical wave. The GIML element is the root element consisting of 14 gesture ements shown in Fig. 3.

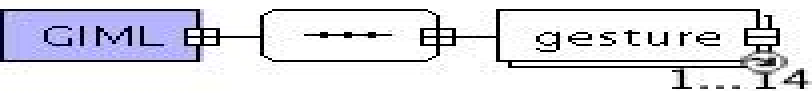

[Fig. 3] The relation between gesture element and GIML emelent 


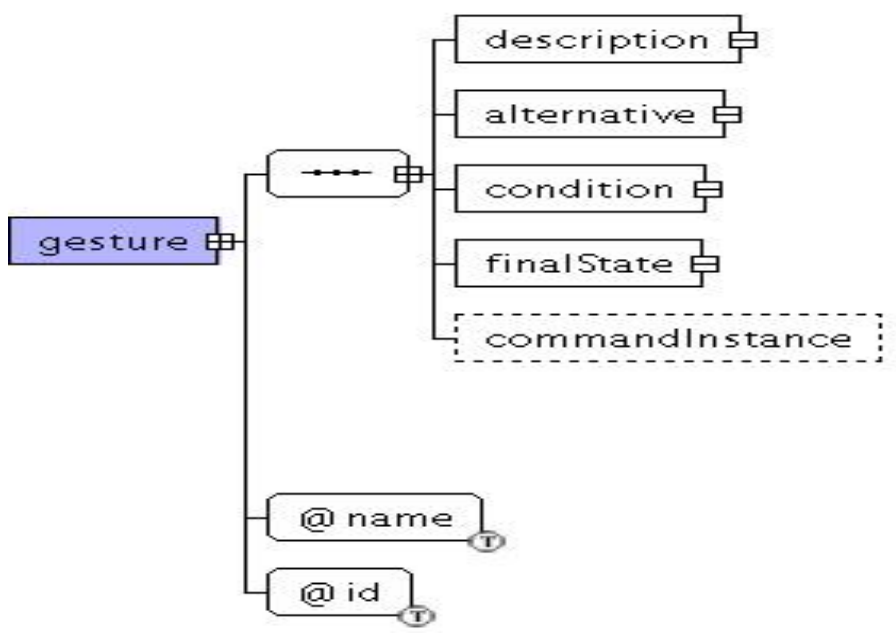

[Fig. 4] The elements of a gesture element

In our definition, the gesture element consists of seven elements: description, alternative, condition, finalStatue, name, id, and commandInstance as shown in Fig. 4. The commandInstance element is optional element, but other six elements are mandatory. The alternative element describes corresponding keyboard or mouse event for the defining gesture. The condition element is used to describe what conditions make the gesture event indicating that the defining gesture is recognized. As shown in Fig. 5, the condition element consists of seven attributes required to decide if the defining gesture occurs. The finalState element contains the gesture related output information to be transferred to the upper gesture-based application when the defining gesture is recognized. This output information shown in Fig. 6 consists of two attributes: a eventName and property giving the positions where the gesture occurs and will be passed to the gesture handler in the gesture-based application.

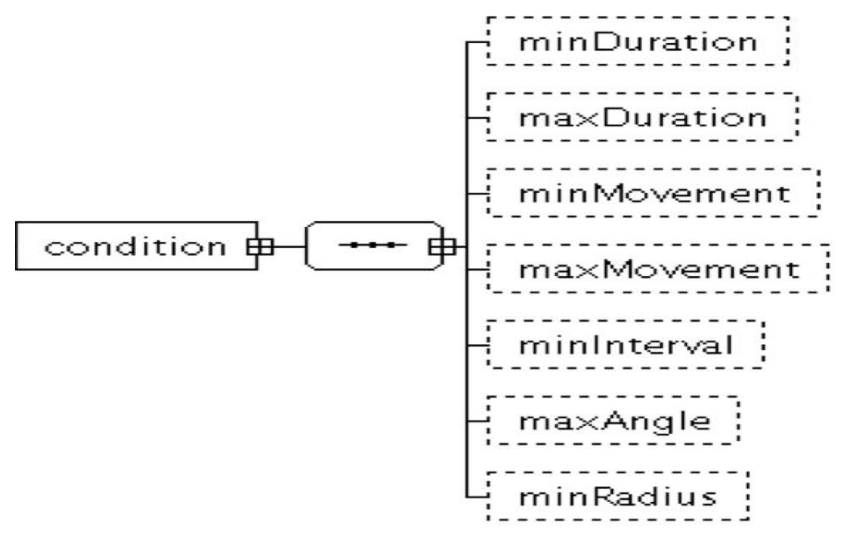

[Fig. 5] The conditions of gesture occurrence 


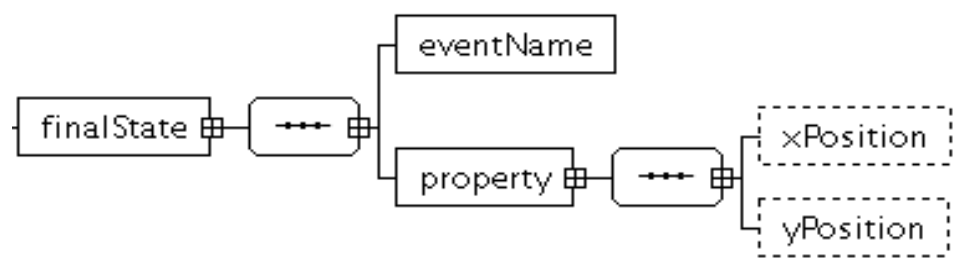

[Fig. 6] The gesture element to be transferred to gesture-based applications

Based on the described $\mathrm{xml}$ schema, we can define gestures to be utilized in gesture recognizer in Fig. 1. The gesture recognizer utilizes gesture definitions described in GIML to implement and check if the occurred movement is correspondent to the defined gesture. Then it issues the corresponding gesture event that contains name and property.

For example, we describe a left and a double tap gesture in Fig. 7 and Fig. 8 respectively.

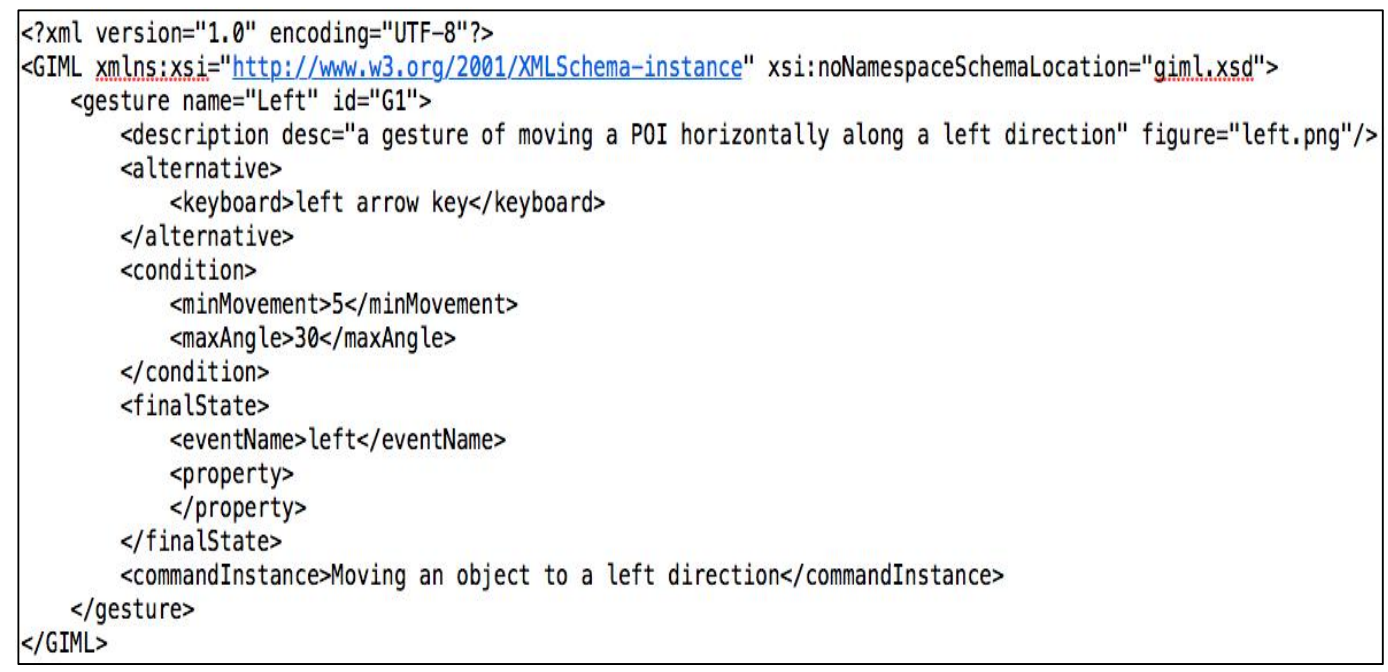

[Fig. 7] The description of left gestureusing GIML 


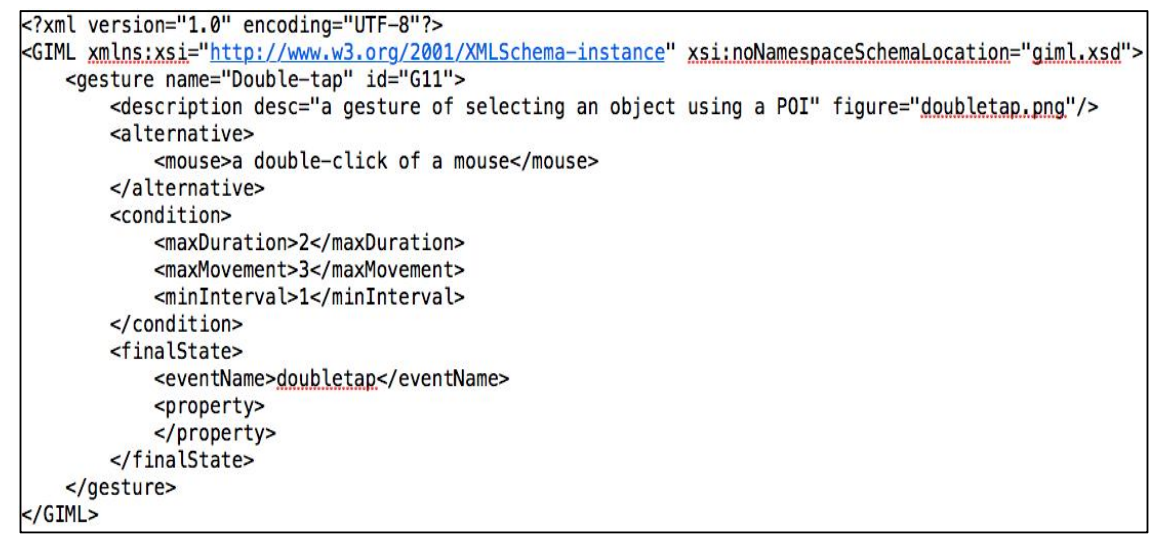

[Fig. 8] The description of double tap gesture using GIML

\section{UGesture Implementation and Evaluation}

In Hammer Library, the double tap gesture generated a tap gesture event as well as a double tap gesture event, which caused a problem because the application developer could not discriminate between two gestures. To resolve this problem, the UGesture platform postpones the invocation of a tap gesture event until it has determined that a double tap gesture has not occurred. We used the test web page shown in Fig. 9 to test the tap gesture and double tap gesture in an element. In the test web page, the tap gesture opened other web page and the double tap gesture enlarged the image. In order to provide the same programming interfaces for mouse, touch, and 3D hand movement detector, we have implemented UGesture platform[13].

In the Hammer Library and the UGesture platform, the tap gesture obtained the same result, i. e., moving to other web page, but the double tab gesture obtained different results. Fig. 10(a) shows the result obtained for the double tab gesture in Hammer library, where the double tap gesture generated a tap gesture event at first. Fig. 10(b) shows the result obtained for the double tap gesture on the UGesture platform where it enlarged the image.

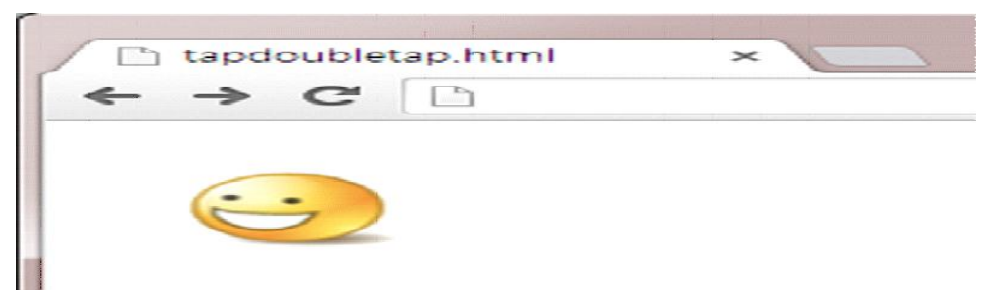

[Fig. 9] The web page to evaluate the double tap gesture 


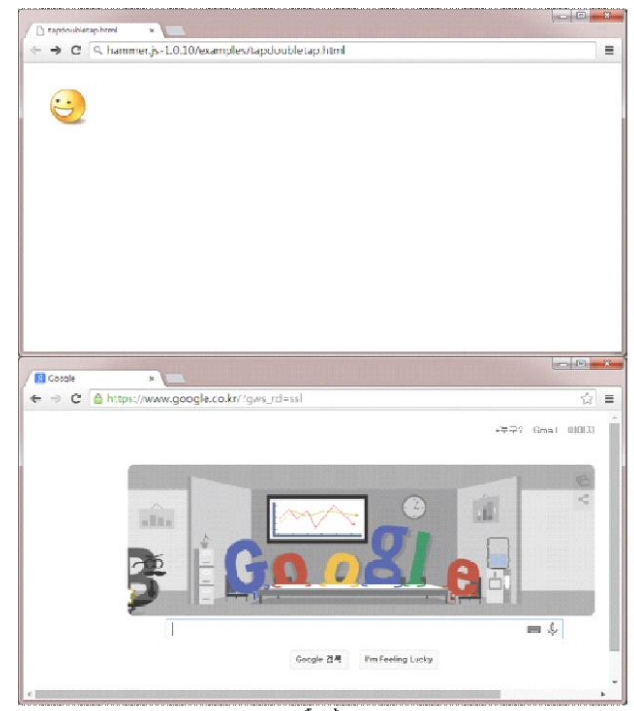

(a)

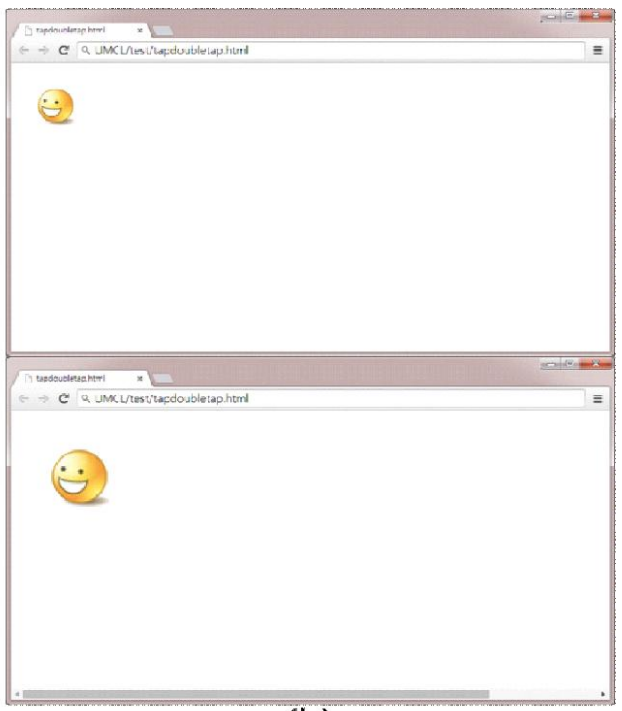

(b)

[Fig. 10] Comparison of double tap gesture on both Hammer and UGesture

In addition, We have performed UGesture platform in terms of element size, distance between two contact points, tap, and double tap gesture[14].

\section{Conclusions}

In this paper, we described briefly the key functions of UGesture platform, and proposed the gesture interface markup language (GIML) based on extensible markup language (XML) to describe gestures independently of the input devices. Finally, we showed the evaluation results in terms of double tap and comparison results of double tap gestures with HammerJS. Since GIML provides constraints necessary to determine which gesture has occurred and information required when UGesture platform interact with the gesture based application, application developers can define the required gesture regardless of input devices and register that to UGesture platform.

As a future work, we will extend the UGesture platform to support GIML to develop a gesture description method which works irrespective of the input devices. 


\section{References}

[1] L. D. Spano, A. Cisternino and F. Paterno, A Composition Model for Gesture Definitions, in Human-Centered Software Engineering, Edited M. Winckler M., P. Forbrig, R. Bernhaupt, Lecture Notes in Computer Science, Berlin (2012), Vol.7623, pp.34-52.

[2] K. Heikkine and J. Porras, UIs - past, present and future, Wireless World Research Forum, White Paper Version 1.0, Dec. (2013)

[3] J. Nielsen, Usability Engineering, Academic Press, ISBN: 0-12-518405-0 (1993)

[4] Micro JavaScript Library, The Grid: Micro JavaScript Library that simplifies you mobile projects, http://www.drupalorg/project/quojs (2016)

[5] Hammer Library: Add touch gestures to you page, http://hammerjs.github.io (2015)

[6] M. Abrams, C. Phanouriou, A. L. Batongbacal, S. M. Williams and J. E. Shuster, UIML: an application-independent XML user interface language, Computer Networks (1999), Vol. 31, No. 1116, pp. 1695-1708.

[7] http://blog.usabilla.com/short-history-computer-user-interface-design/, April 20, (2016)

[8] C. Villamore, D. Willis and L. Wroblewski, Touch Gesture Reference Guide, April 15, (2010)

[9] Safari Web Content Guide - Handling Events https:/developer.apple.com/library/content/documentation/Apple Applications/Reference/SafariWebContent/HandlingEvents/HandlingEvents.html\#//apple_ref/doc/uid/TP40006511-S W1 (2016)

[10] Pointer Events, W3C Recommendation, http://www.w3.org/TR2015/REC-pointerevents-20150224/2015, (2015)

[11] Add touch gestures to your page, http:hammerjs.github.io (2015)

[12] Reach into new worlds, http://www.leapmotion.com, (2015)

[13] E. Song, S. Kim and G. H. Kim, Implementation of Unified Gesture Library for Multiple-Device Environments, in Advances in Computer Science and Ubiquitous Computing, Edited D. S Park, H. C Chao, Y. S. Jeong, J. Park, Lecture Notes in Computer Science, Singapore (2015), Vol.373, pp.535-540.

[14] E. Song and G. H. Kim, A Unified Gesture Platform for Multiple-Device Environment, International Journal of Artificial Intelligence and Applications for Smart Devices (2017), Vol.5, No.2, pp.13-18. 